\title{
Prediction Models for the Elastic Modulus of Fiber-reinforced Polymer Composites: An Analysis
}

\author{
M. A. Islam* and K. Begum \\ Department of Chemical Engineering and Polymer Science, Shahjalal University of Science and \\ Technology (SUST), Sylhet 3114, Bangladesh
}

Received 17 January 2011, accepted in revised form 15 March 2011

\begin{abstract}
An analysis has been done on the existing models for the prediction of the elastic modulus of fiber-reinforced polymer composites (FRPC). The experimental data reported in different specialized research journals have been fitted to the models. It is found the theoretical models such as the Parallel, Series and Halpin-Tsai model, by no means, predict the modulus within an acceptable deviation factor of 0.1. The semi-empirical models such as modified Halpin-Tsai and Bowyer-Bader model, which have one adjustable parameter, and are expressed in terms of volume fraction describe the modulus satisfactorily. In this paper, a mass fraction based model with one adjustable parameter is proposed, which also describe the modulus successfully. The proposed model, being mass fraction-based, is more convenient to work with than any volume-fraction based model, and unlike all other models (theoretical and semi-empirical), it has the potentials to have practical applications in structural material design.
\end{abstract}

Keywords: Fibers; Polymer-matrix composites (PMCs); Short-fiber composites; Mechanical properties; Prediction model.

(C) 2011 JSR Publications. ISSN: 2070-0237 (Print); 2070-0245 (Online). All rights reserved.

doi:10.3329/jsr.v3i2.6881 J. Sci. Res. 3 (2), 225-238 (2011)

\section{Introduction}

Fibers are introduced in polymer compositions in order to improve their mechanical properties. Both synthetic and natural fibers are used for the purpose; although in recent years, polymer-matrix composites with natural fibers have received considerable attention both in the literature and in industrial applications due to their improved mechanical properties, significant processing advantages, low cost and low density [1].

Literature data on fiber-reinforced polymer composites (FRPC) have been accumulating, but reports on theoretical modeling of composition/property relation of these composites are scarce [2-5]. The modulus of elasticity can be considered as one of the most significant mechanical properties of materials for engineering design of structure.

\footnotetext{
* Corresponding author: mislam@sust.edu
} 
This mechanical property of fiber-filled composites are affected by a number of parameters such as fiber type, matrix type, fiber orientation, fiber geometry, volume fraction of the fibers and the degree of interfacial adhesion between the fiber and the polymer matrix [6-10]. The properties of plant fibers, from their part, depend on a number of factors such as the nature of the plant, locality in which they are grown, age of the plant, part of the plant from which they are extracted, extraction methods and so on. Also the mechanical properties of the fibers from a single batch have somewhat statistical distribution. It is very difficult, if not impossible, to take into account all the factors affecting the properties of the fibers and then to propose a model for the estimation of elastic modulus of the FRPC.

Facca et al. [2] applied six micromechanical composite models (theoretical and semiempirical) to predict the properties of the glass, wood, hemp and rice hull fibers reinforced polymer composites. Among all the models tested, the semi-empirical 'Modified HalpinTsai' (MHT) model was found to predict the experimental data satisfactorily.

Kalaprasad et al. [3] also selected a number of micromechanical composite models to predict the properties of the composites with longitudinally as well as randomly oriented fibers. The Hirsch and the Bowyer-Bader (BB) model were found to predict the Young's modulus of the composites with both types of fiber distributions most satisfactorily, whereas both the Rule of Mixture (ROM) or Parallel model and the Inverse Rule of Mixture (IROM) or series model failed to predict it.

Among all the theoretical models, the ROM and the IROM have got the simplest mathematical relations. To apply these models, the modulus of elasticity of the polymer, $E_{p}$, and of the fiber, $E_{f}$, should be known and then the modulus of elasticity of the composite, $E_{c}$, can be calculated for any volume fraction of the fiber in the composition. But those experienced in the field shall admit that these two models, in most cases, do not predict the modulus of elasticity of the composites satisfactorily. The experimental observations and analysis of Facca et al. [2] and Kalaprasad et al. [3] also confirm that. The experimentally observed value of the modulus of elasticity of the composites always lies in between those predicted by the Parallel and Series models.

The Halpin-Tsai (HT) model is also a theoretical model. This model, besides the modulus of elasticity of the polymer, $E_{p}$, and of the fiber, $E_{f}$, includes a geometrical parameter (aspect ratio) of the fiber as well. The model has a complicated mathematical structure, but still failed to predict the observations made by Facca et al. [2] and Kalaprasad [3]. The semi-empirical MHT model, however, predicted the experimental results of Facca et al. [2] satisfactorily. On the other hand, the experimental results of Kalaprasad et al. [3] were described satisfactorily by another semi-empirical model namely Bowyer-Bader (BB) model.

The modulus of elasticity of FRPC in all the models tested by Facca et al. [2] and Kalaprasad [3] is expressed as a function of the volume fraction of the fiber, $v_{f}$. Virtually, the experiments are usually designed in terms of mass fraction, and the volume fraction is calculated based on the density data (available in the Literature) of the polymer and the fiber. Facca et al. [2] have shown that the density of the polymer-fiber composite is not an 
additive quantity with the contribution of the components proportional to their volume fraction. This will mean that although the mass fraction is known, the volume fraction remains unknown. For prediction purposes, however, one has to know the volume fraction. Then one has to conduct experiments and collect the density data of the composites for different mass fractions and find the volume fraction versus mass fraction relationship.

From the literature data [2-4], it has become evident that the theoretical models (which do not contain any adjustable parameter) fail to predict the modulus of elasticity of FRPCs, and to predict the modulus satisfactorily, one has to apply a relation with at least one adjustable parameter, which provide the model with semi-empirical nature. As there is no escape from empirical relation, it is easier to use some predictive model expressed in terms of mass fraction instead of volume fraction.

Recently Mirbagheri et al. [11] conducted intensive research on hybrid composites consisting of ternary mixture of wood flour, kenaf fiber and polypropylene, and found that the ROM could successfully describe the modulus of elasticity of the polymer composites. Fu et al. [12], on the other hand, applied two approaches, namely ROM and LAA (Laminate Analogy Approach) to describe the elastic modulus of a ternary mixture of particle-fiber-polymer. The authors observed that the experimentally observed value of the elastic modulus is, to some extent, described by the LAA approach, but is much higher than that predicted by ROM. Conversely, in binary mixture of polymer and fiber, the elastic modulus of the composite is found to be much lower than that predicted by the ROM [2, 3].

The present work deals basically with two-component systems consisting of polymer and fibers, and the probable variation of the modulus of elasticity of a given component in the composite structure from that in the pure state has been ignored in the analysis. The purpose of the present work is (1) to make a discussion on the applicability of the ROM and IROM, HT, MHT and BB model as the most discussed ones among the mentioned models in predicting the Young's modulus of some FRPCs, (2) To test whether the theoretical models (without any adjustable parameter) namely, ROM, IROM and HT model could predict the modulus within a tolerance range of deviation, (3) to propose a simple model in terms of mass fraction with one adjustable parameter to describe some experimental data of different fiber-reinforced polyethylene and polypropylene composites available in the literature[1, 2, 5, 13], and to compare its predictability with that of MHT and BB models, which also contain one adjustable parameter, and (4) finally to discuss in brief the perspective of the proposed model in serving the real purpose of prediction i.e. whether one could rely on the fitted value of the adjusted parameter to be equally valid for untested composition of the same material.

It has been found that none of the models without adjustable parameters can predict the modulus of elasticity satisfactorily. Using small number of literature data, it has been observed that the models with an adjustable parameter, expressed in terms of volume or mass composition could describe the experimental data successfully. It is recommended, however, that more data is necessary to validate the models and the fitted value of the 
adjustable parameter would then be used to predict the modulus of elasticity of untested compositions.

\section{Most Discussed Models and a Newly Proposed One}

A number of models are available in the literature [2, 3, 14-16] for the prediction of the Young's modulus of the FRPCs. Among them, the following have comparatively simpler mathematical structure and are the most frequently discussed ones: (1) ROM or parallel model (2), IROM or series model, (3) HT model, (4) MHT model and (5) BB model. In the following sections, the applicability of the models will be discussed. Also a new model with empirical structure similar to that of the BB model, but expressed in terms of mass fraction, will be proposed.

\subsection{Rule of mixture (ROM) or parallel model}

The simplest available model to predict the elastic modulus of a composite material is the rule of mixtures (ROM) [14]. The ROM equation for the Young's modulus is:

$$
E_{c}=E_{f} v_{f}+E_{p} v_{p} \quad \text { with } \quad v_{f}+v_{p}=1
$$

where the subscripts $c, f$ and $p$ stand for composite, fiber and polymer matrix respectively, $E_{c}, E_{f}$ and $E_{p}$, are the elastic moduli, and $v_{f}$, and $v_{p}$, are the volume fractions.

Eq. (1) can be theoretically derived for a system, in which the fibers are aligned along $z$-axis and propagate through the whole length of the body as shown in Fig. 1. A force (equivalent to stress $\sigma_{z}$ ) is acting along the z-axis. Then the modulus of elasticity along $z$ axis is given by Eq. (1) [17]. This equation is very attractive with superficially interesting features: a) This equation is apparently valid for $v_{f} \in(0,1)$ and b) for $v_{f}=0, E_{c}=E_{p}$, and for $v_{f}=1, E_{c}=E_{f}$.

Eq. (1) is derived for long fibers (The fiber length is equal to the height of the object), but in most cases, attempts are made to apply it for composites with short-fibers distributed randomly. It is also ignored that beyond certain value of $v_{f}$ (far below unity), the composite loses its integrity and Eq. (1) cannot be applied for high value of $v_{f}$.

There is a popular perception that Eq. (1) is a theoretical model as it can be derived for the distribution of fibers along the axis of elongation. For short-fiber reinforced composites, however, the equation is not valid at all. The polymer-fiber composite is a heterogeneous mixture with well-distinguished interface, and the property of the fiber is imparted to the matrix through this interface. The composite is not a homogeneous solution that an additive law will be expected to hold true for the property of the polymer matrix and the fiber equation. Therefore, it is not unusual that the experimental data of the modulus of elasticity versus volume fraction is not described by the model represented by Eq. (1) as reported in the literature [2, 3]. 


\subsection{Inverse rule of mixture (IROM) or series model}

The series model [15] is represented by the following relation:

$$
E_{c}=\frac{1}{v_{f} / E_{f}+\left(1-v_{f}\right) / E_{p}}
$$

One could expect that Eq. (2) would be derived if the load (equivalent to the stress $\sigma_{x}$ or $\sigma_{y}$ ) is applied to the object in Fig. 1 along $x$ or $y$ axis. This is, however, not the case. For the derivation of Eq. (2), the fiber and polymer material are arranged in a manner as shown in Fig. 2, and a load is applied along $y$ - direction and then the Eq. (2) is derived [17]. If the load is applied along $x$ - or $z$ - axis to the object shown in Fig. 2, the ROM or Eq. (1) will be obtained. Such an arrangement, however, is not realistic for a composite, as this does not ensure adhesion between the polymer and the fiber. This is not a composite; rather separate elements arranged in series. Thus, Eq. (2) is not a theoretical model for short-fiber reinforced polymer composites. The IROM model (Eq. 2) also fails to describe the experimental data on the modulus of elasticity of FRPC versus volume fraction of the fibers [2, 3].

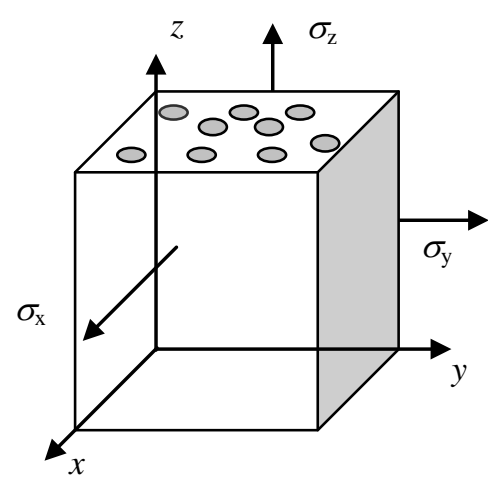

Fig. 1. Distribution of the fibers oriented along $z$-axis and propagating through the whole length of the cube.

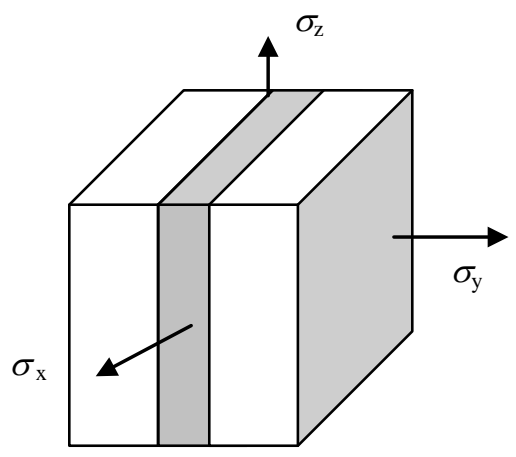

Fig. 2. Tri-dimensional view of a 'hypothetical'/model arrangement of fibers and polymer matrix: parallel arrangement as viewed along $z$ - and $x$-axis, and series arrangement as viewed along $y$-axis.

\subsection{Halpin-Tsai (HT) and modified Halpin-Tsai (MHT) model [2, 16]}

The HT model (Eq. 3) has a more complicated mathematical structure than that of the ROM or IROM. In this model, in predicting the elastic modulus of the composites, besides the modulus of elasticity and volume fraction of the components, the aspect ratio (ratio of the geometric dimensions) of the fiber is also taken into consideration (Eq. (3)). 


$$
E=E_{p}\left(\frac{1+\xi \eta v_{f}}{1-\eta v_{f}}\right) \text { with } \eta=\frac{E_{f} / E_{p}-1}{E_{f} / E_{p}+\xi} \quad \text { and } \xi=\frac{2 L}{T} \quad \text { or } \xi=\frac{2 L}{D}
$$

where $L$ refers to the length of the fiber, and $T$ or $D$ is the thickness or diameter of the fiber. The Eq. (3) does not have any adjustable parameter and knowing the properties of the components, the modulus of elasticity of the composite could be calculated. For $\xi \rightarrow 0$, Eq. (3) is reduced to Eq. (2) representing the IROM or Series Model and for $\xi \rightarrow \infty$, Eq. (3) is reduced to Eq. (1) representing the ROM or Parallel Model. Thus, the HT model is a more general model. In spite of the complicated mathematical structure, the HT also fails to predict the elastic modulus of polymer-fiber composites satisfactorily. If the term $\xi$ is replaced by an adjustable parameter $\xi_{a d}$, then the HT model is reduced to Eq. (4).

$$
E=E_{p}\left(\frac{1+\xi_{a d} \eta v_{f}}{1-\eta v_{f}}\right) \text { with } \eta=\frac{E_{f} / E_{p}-1}{E_{f} / E_{p}+\xi_{a d}}
$$

Eq. (4) is known as modified Halpin-Tsai (MHT) model and in this form it predicts satisfactorily the elastic modulus of fiber-reinforced polymer composites [2-4]. The parameter $\xi$ in HT model accounts for the packing arrangement and the geometry of the fibers. The adjustable parameter $\xi_{a d}$ in MHT model, however, does not have any physical significance. The value of the parameter $\xi_{a d}$ is adjusted in such a way that the experimental $E_{c}$, vs. $v_{f}$, data fit to Eq. (4) and hence, the MHT model does not have credibility more than an empirical relation.

\subsection{Bowyer-Bader (BB) model}

The Bowyer-Bader (BB) model [3] is represented by the following relation:

$$
E_{c}=E_{m} v_{m}+\alpha_{v} E_{f} v_{f} \quad \text { with } \quad \alpha_{v}=k_{1} \cdot k_{2}
$$

where $k_{1}$ and $k_{2}$ are the fiber orientation and the fiber length factor respectively, and the parameter $\alpha_{v}$ is the overall reinforcing factor. In fitting the experimental $E_{c} v s . v_{f}$ data to Eq. (5), one has to adjust the value of the product $k_{1} \cdot k_{2}$. It is difficult to determine the value of $k_{1}$ and $k_{2}$ separately. In literature [3], some formula is used to calculate the individual value of $k_{1}$, but such formula merely has much solid theoretical ground. Thus, the BB model is with one adjustable parameter, $\alpha_{v}$, and has got much simpler mathematical structure than that of MHT model. For $\alpha_{v}=1$, Eq. (5) is converted into Eq. (1) representing the parallel model. The parameter $\alpha_{v}$ expresses to what extent the modulus of elasticity of the fiber contribute to the modulus of elasticity of the composite. The BB model satisfactorily describes the elastic modulus of a FRPC [3].

\subsection{A Mass fraction-based simple (MFS) model}

A simple empirical model could be formulated in terms of mass fraction in order to predict the modulus of elasticity of FRPC. The MFS model may be represented by Eq. (6) 


$$
E_{c}=E_{p} x_{p}+\alpha_{m} E_{f} x_{f} \quad \text { with } \quad x_{p}+x_{f}=1
$$

where $x$ is the mass fraction of the components and $\alpha_{m}$ is the reinforcing factor in mass fraction based empirical relation. This model has got mathematical structure similar to that of BB model (Eq. 5), but unlike all other mentioned models, in this model the modulus of elasticity of FRPC is expressed in terms of mass fraction. If the Eq. (6) could describe the elastic modulus of the composites, it would serve the practical purposes of structural material design much better than other models, avoiding difficulties in the conversion of mass fraction to volume fraction.

\section{Illustration of the Model Prediction}

Before going for validation of the models, one has to be very cautious about the quality of data being used. For the validation of the models, the first two parameters that are essential to be known are the modulus of elasticity of the polymer and the fibers. It is a well-known fact that the polymer properties vary in a wide range depending on the chemical composition, chain-branching, molecular mass distribution and the processing parameters. Therefore, any value of $E_{p}$ found in literature could, by no means, be considered an acceptable one without reservations. The properties of natural fibers also vary in a wide range. The variation is caused by the fluctuation in environmental conditions (moisture, soil, temperature etc) in the regions, in which the fibers grow and also on the processing methods. Even in the same batch, the properties of fibers have statistical distribution. For this reason, it must be defined first in what range the prediction would satisfy the requirements to the design of structural material.

\section{Tolerable deviation from the values predicted by the models}

Let us assume that the coefficient of deviation in the value of the parameters $E_{p}$ and $E_{f}$ are respectively $\gamma_{p}$ and $\gamma_{f}$; i.e. $\gamma_{p}=\Delta E_{p} / E_{p}$ and $\gamma_{f}=\Delta E_{f} E_{f}$. The deviation in the value of the parameters $E_{p}$ and $E_{f}$ will definitely reflect in the deviation of the estimated value of $E_{c}$.

\section{Equation for error calculation in ROM or parallel model [18]:}

Following Eq. (1), we have

$$
d E_{c}=v_{f} d E_{f}+v_{p} d E_{p}
$$

Then the deviation in the elastic modulus of the composite, $\Delta E_{c}$, is given by

$$
\Delta E_{c}=v_{f} \Delta E_{f}+v_{p} \Delta E_{p}
$$

Substituting $\Delta E_{f}$ and $\Delta E_{p}$ with their equivalents, the Eq. (8) is reduced to Eq. (9).

$$
\Delta E_{c}=\gamma_{f} v_{f} E_{f}+\gamma_{p} v_{p} E_{p}
$$


For every volume fraction $v_{f}$, the error $\Delta E_{c}$ in the estimation of $E_{c}$ will be determined from Eq. (9).

\section{Equation for error calculation in IROM or Series model [18]:}

Following Eq. (2), we have

$$
\frac{d E_{c}}{E_{c}}=E_{c}\left[\frac{v_{f}}{E_{f}} \cdot \frac{d E_{f}}{E_{f}}+\frac{v_{p}}{E_{p}} \cdot \frac{d E_{p}}{E_{p}}\right]
$$

Then the deviation in the elastic modulus of the composite, $\Delta E_{c}$, is given by

$$
\frac{\Delta E_{c}}{E_{c}}=E_{c}\left[\frac{v_{f}}{E_{f}} \cdot \frac{\Delta E_{f}}{E_{f}}+\frac{v_{p}}{E_{p}} \cdot \frac{\Delta E_{p}}{E_{p}}\right]
$$

Substituting $\Delta E_{p}$ and $\Delta E_{f}$ with their equivalents, Eq. (11) is reduced to Eq. (12):

$$
\frac{\Delta E_{c}}{E_{c}}=E_{c}\left[\gamma_{f} \frac{v_{f}}{E_{f}}+\gamma_{p} \frac{v_{p}}{E_{p}}\right]
$$

For every volume fraction $v_{f}$, the error in the estimation of $E_{c}$ will be determined from Eq. (12).

For simplicity, let us assume that $\gamma_{p}=\gamma_{f}=0.1$. Then for both Eq. (9) and Eq. (12) (corresponding to parallel and series model), $\Delta E_{c}=0.1 E_{c}$. This would mean that if the experimentally obtained value of the modulus of elasticity of the composite, $E_{\text {exp }}$, with a given composition lies within the range of $(1.0 \pm 0.1) E_{c}$, the prediction may be considered satisfactory. In the similar manner, the formula for acceptable deviation of the predicted value can be estimated for the HT model.

\section{Validation of ROM, IROM and HT Model}

These three models do not have any adjustable parameter. If the experimental modulus of elasticity $E_{\text {exp }} v s$. fiber volume fraction $v_{f}$ data is plotted and the curve lies in the area surrounded by the prediction curves $0.9 E_{c}$ and $1.1 E_{c}$, then the prediction might be considered satisfactory. This procedure of validation is applied to some polymer fiber composites such as High density polyethylene (HDPE)-E-glass, HDPE-Hardwood A, HDPE-Hardwood B, HDPE-Hemp, HDPE-Rice hulls, Low density polyethylene (LDPE)Sisal and Polypropylene (PP)-Flax system. The data were collected from the literature [2$4,13]$. The figures were drawn (only two of these are shown) and it was found that in all cases (except HDPE-E-glass system), the experimental curve, $E_{\text {exp }}$, lay far apart from the area surrounded by the curves $0.9 E_{c}$ and $1.1 E_{c}$ as predicted by the ROM, IROM and HT model. Fig. 3 represents the experimental data (collected from literature) and also the 
curves representing the modulus of elasticity of HDPE-Hardwood A fiber composites as predicted by the HT (Eq. 3) and the IROM model (Eq. 2). The indices 0.9 and 1.1 denote the multiple factor to the estimated $E_{c}$. It is evident from the Fig. 3 that neither IROM nor HT could predict the Young's modulus $E_{c}$. Such was the picture with the prediction by the ROM, IROM and HT model on all mentioned systems excluding the HDPE-E-glass fiber composite.

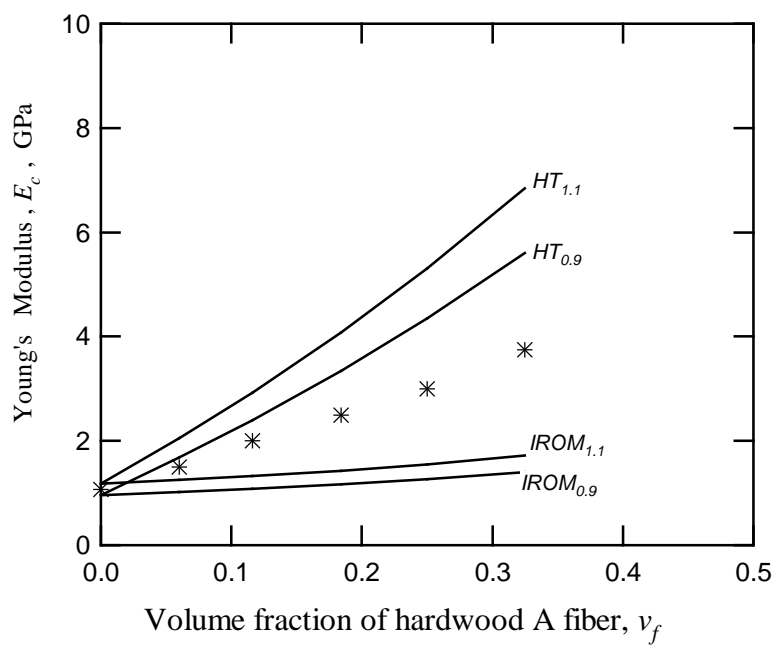

Fig. 3. Young's modulus $E_{c}$ vs. volume fraction of the fiber, $v_{f}$, in HDPE-Hardwood A composite. The symbol * represents the experimental data [2]. The interpretation of the symbols is given in the text.

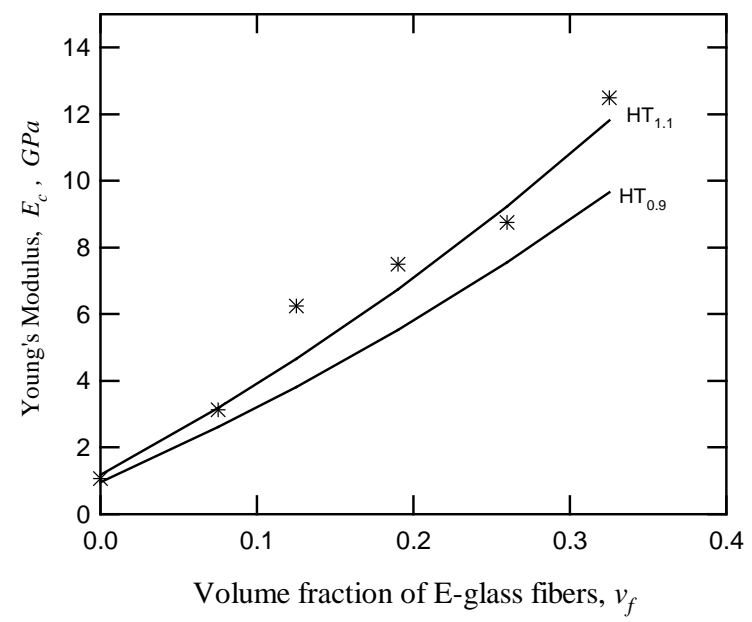

Fig. 4. Young's modulus $E_{c}$ vs. volume fraction of the fiber, $v_{f}$, in HDPE-E-Glass fiber composite. The symbol * represents experimental data [2]. The interpretation of the symbols is given in the text. 
Fig. 4 presents the experimental data of HDPE-E-glass fiber composites (collected from Literature) and the prediction of HT model. In this case also the modulus of elasticity predicted by the ROM and IROM lies far apart from the experimentally observed modulus of elasticity (not shown in the figure). The $\mathrm{HT}_{0.9}$ and $\mathrm{HT}_{1.1}$ curves, however, partially cover the experimental data, and this is the 'best' prediction among the seven systems studied. Thus, it is hard to rely on the ROM, IROM or HT model to predict the modulus of elasticity of a polymer-fiber composite. Facca et al. [2] have also excellently demonstrated these failures.

\section{Validation of the MHT, BB and MFS Model}

Each of these three models contains an adjustable parameter. Assigning some constant value to $E_{p}$ and $E_{f}$, the value of the parameter ( $\xi_{a d}, \alpha_{v}$ or $\alpha_{m}$ ) is to be adjusted in such a manner that the $E_{c}-E_{p} v s$. volume fraction, $v_{f}$, data fits to the MHT (Eq. 4) and BB (Eq. 5) model, and the $E_{c}-E_{p}$ vs. mass fraction, $x_{f}$, data to the MFS (Eq. 6) model. The result of the validation of the models is presented in Figs. 5, 6 and 7, and the value of the adjusted parameter is presented in Table 1.

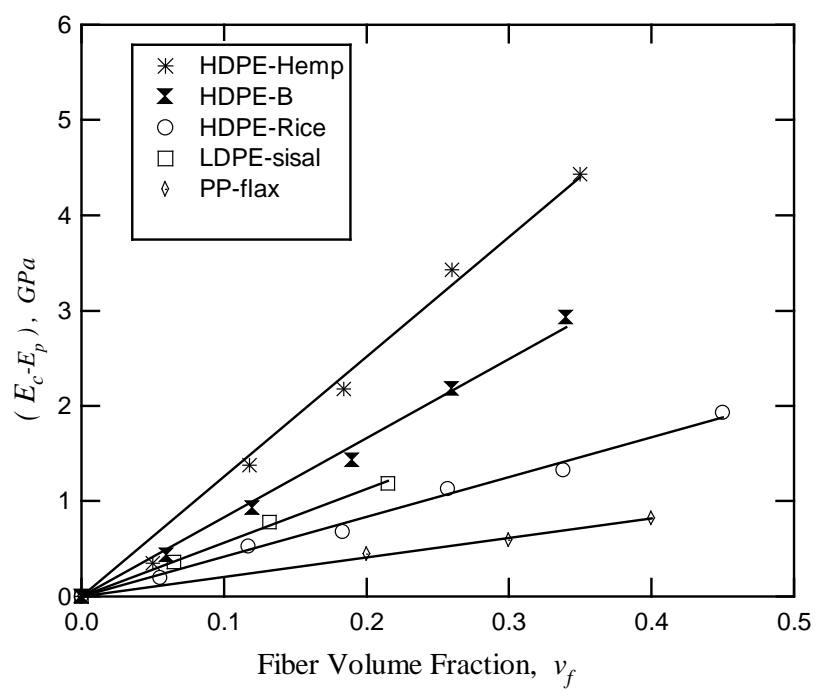

Fig. 5. $\left(E_{c}-E_{p}\right)$ vs $v_{f}$ data for HDPE-Hemp, HDPE-Hardwood B, HDPE-Rice hulls, LDPE-Sisal and PP-Flax fiber composites fitted to BB model. The data were collected from ref. [2-4].

Table 1 represents the assigned value to $E_{p}$ and $E_{f}$ of different polymer-fiber system and the value of the corresponding fitted parameter of the MHT, BB and PM models. The value of $\xi_{a d}$ found in this treatment differs a bit from that reported by Facca et al. [2]. The reason for such difference lies probably in the error in the collection of secondary data from the figures in Facca et al. [2]. The volume fraction and the corresponding mass fraction of only some of the compositions in Table 1 were available in the literature. For 
this reason, the data of only few of the compositions were treated with all the three models. Some data have been treated only with MHT and BB model (which are volume fraction based) and some data- only with MFS model (which is mass-fraction based). For this reason, some of the cases in Table 1 remained empty. In spite of the deficiency of data, from a glance on the curves in Figs. 5, 6 and 7 and the data in Table 1, it may be concluded that all the three models could successfully describe the modulus of elasticity $E_{c}$ of FRPCs. Facca et al. [2] successfully fitted their experimental data to MHT model. Kalaprasad et al. [3] and Joseph et al. [13] fitted their data to BB model. It is worth

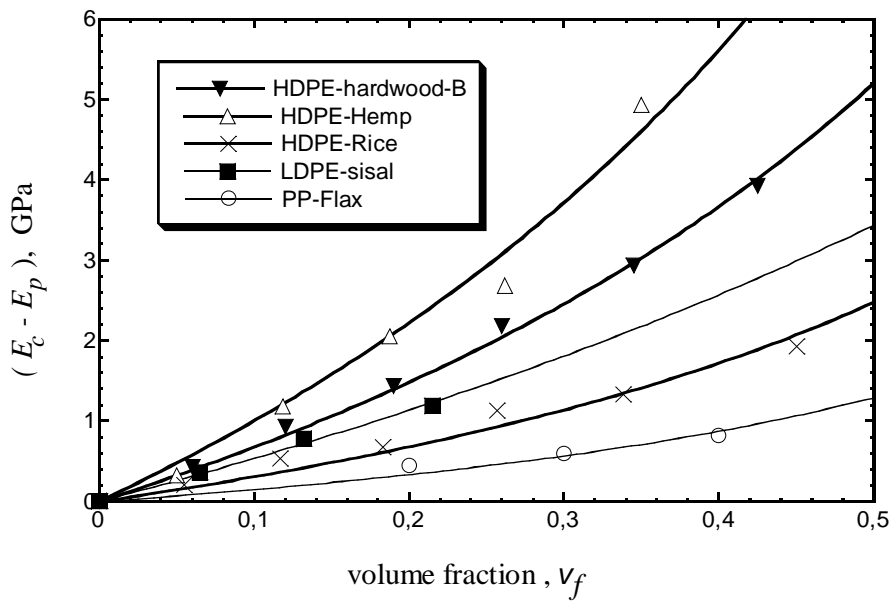

Fig. 6. $\left(E_{c}-E_{p}\right)$ vs $v_{f}$ data for HDPE-Hemp, HDPE-Hardwood B, HDPE-Rice hulls, LDPE-Sisal and PP-Flax fiber fitted to MHT model. The data are collected from ref. [2-4].

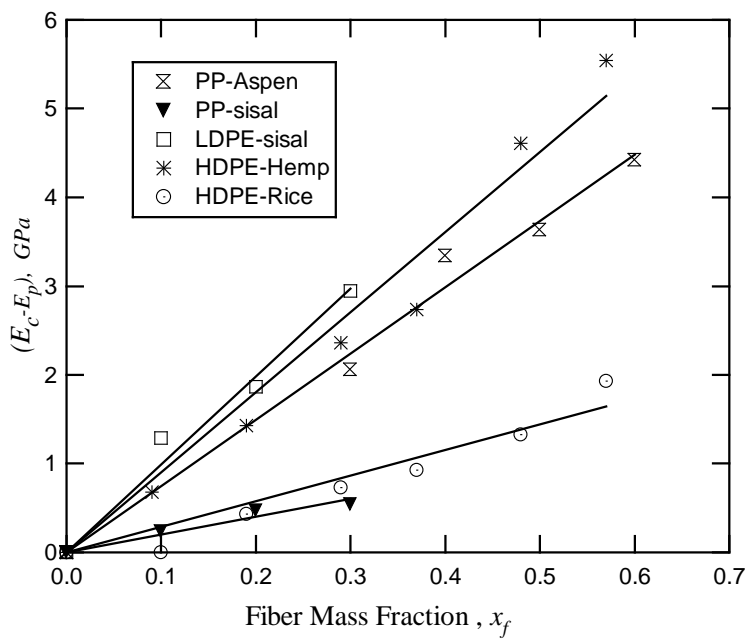

Fig. 7. $\left(E_{c}-E_{p}\right)$ vs mass fraction, $x_{f}$ data for PP-Aspen, PP-Sisal, HDPE-Hemp and HDPE-Rice hull fibers fitted to MFS model. The data are collected from ref. [1, 2, 5]. 
mentioning that the experimental $E_{c} v s . v_{f}$ data of none of the polymer-fiber system described in Table 1 fit to the theoretical models (ROM, IROM and HT). The illustration in Figs. 5, 6 and 7 and the data in Table 1 categorically show that the data of all the polymer-fiber composites under discussion in this paper could be described by any one of three models, namely MHT, BB and MFF models. The MFS model being the only mass fraction based model among the three will serve the practical purposes of composite design.

Table 1. Modulus of elasticity and the value of fitted parameters of the MHT (Eq. 4), BB (Eq. 5) and the MFS model (Eq. 6).

\begin{tabular}{lllllll}
\hline Composition & $\begin{array}{l}E_{p} \\
(\mathrm{GPa})\end{array}$ & $\begin{array}{l}E_{f} \\
(\mathrm{GPa})\end{array}$ & $\begin{array}{l}\xi_{a d} \\
(\text { Eq. 4) }\end{array}$ & $\begin{array}{l}\alpha_{v} \\
\text { (Eq. 5) }\end{array}$ & $\begin{array}{l}\alpha_{m} \\
\text { (Eq.6) }\end{array}$ & $\begin{array}{l}\text { Data } \\
\text { source }\end{array}$ \\
\hline HDPE-E-glass & 1.07 & 72.0 & $42 \pm 4.0$ & $0.48 \pm 0.02$ & $0.24 \pm 0.02$ & {$[2]$} \\
HDPE -Hardwood A & 1.07 & 32.7 & $5.5 \pm 0.2$ & $0.30 \pm 0.01$ & $0.19 \pm 0.02$ & {$[2]$} \\
HDPE -Hardwood B & 1.07 & 32.7 & $6.2 \pm 0.1$ & $0.29 \pm 0.01$ & $0.21 \pm 0.01$ & {$[2]$} \\
HDPE -Hemp & 1.07 & 69.0 & $8.9 \pm 0.4$ & $0.19 \pm 0.01$ & $0.15 \pm 0.01$ & {$[2]$} \\
HDPE -Rice hulls & 1.07 & 22.0 & $2.0 \pm 0.1$ & $0.25 \pm 0.01$ & $0.17 \pm 0.01$ & {$[2]$} \\
LDPE-Sisal & 0.14 & 10.75 & $69 \pm 4.0$ & $0.53 \pm 0.01$ & $0.93 \pm 0.05$ & {$[3,13]$} \\
Polypropylene-Aspen & 2.08 & 10.9 & - & - & $0.87 \pm 0.02$ & {$[5]$} \\
Polypropylene-Sisal & 0.498 & 10.75 & - & - & $0.23 \pm 0.01$ & {$[13]$} \\
Polystyrene-Sisal & 0.39 & 10.75 & - & - & $0.25 \pm 0.02$ & {$[13]$} \\
Polypropylene-Flax & 0.49 & 35 & $1.8 \pm 0.1$ & $0.072 \pm 0.001$ & - & {$[4]$} \\
\hline
\end{tabular}

\section{Scope of the MFS Model}

It is highly inspiring that a simple first order relation in terms of mass fraction, $x_{f}$, can describe the $E_{c}$ vs. $x_{f}$ relationship for FRPCs. The model has been validated, however, with small number of data. With the accumulation of data in the literature, it may so happen that the linear equation fails to describe the $E_{c} v s . x_{f}$ relationship for many FRPCs. There will be no harm then to introduce a higher order relation in terms of mass fraction. There should be an end, however, to proposing empirical relation with increasing number of adjustable parameters and subsequent validation. The success of a model virtually lies in the prediction of properties of untested composition, process or phenomenon. Then arises the very question, in what confidence range could the MFS model predict the elastic modulus of FRPCs, which are prepared based on some other brands of polymers and fibers. Could it predict within the range of $(1.0 \pm 0.1) E_{c}$ accepting the value of the $\alpha_{m}$ equal to that described in Table 1? If one can not use the value of $\alpha_{m}$ as a characteristic parameter for a given polymer-fiber system to estimate the elastic modulus, such empirical relation will remain only in papers and will not serve any real purpose. 
The value of $\alpha_{m}$ in this paper has been determined by fitting the $\left(E_{c}-E_{p}\right) v s . x_{f}$ data for a given polymer-fiber system with definite value of the modulus of elasticity $E_{p}$ and $E_{f}$ for the polymer and the fiber respectively. The modulus of elasticity of a polymer may vary in a wide range depending on the molecular mass, branching, preparation and processing condition and so on. The modulus of elasticity of natural fiber also varies in a wide range. Through extensive experimental work, the dependence of $\alpha_{m}$ on varying polymer and fiber properties should be found, and only then the parameter $\alpha_{m}$ will appear to be characteristic of a given polymer-fiber system, and the model will assume the status of prediction equation. Until then the model with the parameter-value equal to that mentioned in Table 1 will serve only the purpose of orientation about the ranges of the modulus of elasticity of a given composite system.

\section{Conclusions}

a. Theoretical models such as ROM (Parallel), IROM (Series) and Halpin Tsai models totally fail to predict the Young's modulus of fiber-reinforced polymer composites.

b. Semi-empirical models such as Modified Halpin-Tsai (MHT) and Bowyer-Bader (BB) model (which are expressed in terms of volume fraction and with one adjustable parameter) successfully describe the Young's modulus of a number of fiber-reinforced polymer composites. The adjustable parameter in the MHT model is an empirical parameter to fit the experimental data to the model and thus, it does not have any physical significance. The adjustable parameter in the BB model is also an empirical parameter, but has got its physical significance as the degree of contribution of the fiber to the composite.

c. The proposed MFS model with one adjustable parameter and expressed in terms of mass fraction also successfully describes the Young's modulus of a number of fiber-reinforced polymer composites. The adjustable parameter in this model has got its physical significance as the degree of contribution of the fiber to the composite.

d. The proposed model being mass fraction based is more convenient to work with than a volume fraction based model, and unlike all other models (theoretical and semi-empirical), it has the potentials to have practical applications in structural material design.

e. The fitted value of the adjustable parameter of the MHT, BB and the proposed one, MFS, still can not be the characteristic parameter of the polymer-fiber systems under investigation. More experimentation is required with varying polymer and fiber properties in order to find a representative value of the adjusted parameter, and the value so obtained only can represent the corresponding polymer-fiber system.

\section{Appendix: List of abbreviations}

BB (Bowyer-Bader); FRPC (Fiber reinforced polymer composite); HDPE (high density polyethylene); HT (Halpin-Tsai); IROM (Inverse Rule of Mixture); LAA (Laminate Analogy 
Approach); LDPE (low density polyethylene); MFS (Mass fraction-based simple); MHT (Modified Halpin-Tsai); ROM (Rule of Mixture); $E_{c}$ (Young's modulus of the composite); $E_{\exp }$ (Experimental Young's modulus of the composite); $E_{f}$ (Young's modulus of the fiber); $E_{p}$ (Young's modulus of the polymer); $v_{f}$ (volume fraction of the fiber); $v_{p}$ (volume fraction of the polymer); $\alpha_{m}$ (adjustable parameter for mass fraction based model); $\alpha_{v}$ (adjustable parameter for Bowyer-Bader model); $\xi_{a d}$ (adjustable parameter for Halpin-Tsai equation); $\sigma$ ( stress applied to the composite).

\section{References}

1. P. V. Joseph, K. Joseph, and S. Thomas, Compos. Sci. Technol. 59, 1625 (1999). doi:10.1016/S0266-3538(99)00024-X

2. A. G. Facca, M. T. Kortschot, and N. Yan, Compos. Part A 37, 1660 (2006). doi:10.1016/j.compositesa.2005.10.006

3. G. Kalaprasad, K. Joseph, and S. Thomas, J. Mater. Sci. 32, 4261 (1999). doi:10.1023/A:1018651218515

4. L. Peponi, J. Biagiotti, M. Kenny, and I. Mondragon, Polym. Composite 29, 321 (2008). doi:10.1002/pc.20386

5. Y. Xue, D. R. Veazie, C. Glinsey, M. F. Horstemeyer, and R. M. Rowell, Compos. Part B 3, 152 (2007). doi:10.1016/j.compositesb.2006.07.005

6. M. J. Folkes, Short fiber reinforced thermoplastics composites (Wiley, New York, 1982).

7. R. P. Sheldon, Composite polymeric materials (Applied Science Publishers, London, 1982)

8. A, Kelly and N. H. Macmillan, Strong solids (Oxford: Clarendon Press, 1986).

9. W. Brostow and R. D. Corneliussen, Failure of plastics (New York: Hanser, 1986).

10. D. Hull, An introduction to composite materials (London: Cambridge University Press, 1981).

11. J. Mirbagheri, M. Tajvidi, I. Ghasemi, and J. C. Hermanson, Iran. Polym. J. 16(4), 271 (2007).

12. S. Y. Fu, G. Xu, and Y.W. Mai, Compos. Part B 3, 291 (2002). doi:10.1016/S1359-8368(02)00013-6

13. K. Joseph, R. D. T. Filho, B. James, S. Thomas, and L. H. de Carvalho, R. Bras. Eng. Agric. Ambiental, Campina Grande 3, 367 (1999).

14. G. C. Sih, A. Carpinteri, and G. Surace, Advanced technology for design and fabrication of composite materials and structures (Kluwer Academic publishers, Dordrecht, 1995).

15. R. M. Jones, Mechanics of composite material (Hemisphere Publishing Corporation, New York, 1975).

16. J. C. Halpin and J. I. Kardos, Polym Eng Sci. 16, 344 (1976). doi:10.1002/pen.760160512

17. F. L. Matthews, R. D. Rawlings. Composite Materials: Engineering and Science (Chapman \& Hall, London 1994).

18. S. Akhnazarova and V. Kafarov, Experiment optimization in Chemistry and Chemical Engineering (Mir Publishers, Moscow, 1982). 